\title{
Por una geografía política sistemática: las áreas nucleares y el sistema de Estados europeo
}

\author{
María LOIS \\ Facultad de Ciencias Políticas y Sociología \\ Universidad Complutense de Madrid
}

\section{REFERENCIA NORMALIZADA}

Lois, María (2014) "Comentario. Por una geografía política sistemática: las áreas nucleares y el sistema de Estados europeo”. Geopolítica(s). Revista de estudios sobre espacio y poder, vol. 5, núm. 1, 127-134.

\section{Introducción}

Escribir un comentario sobre el artículo que nos ocupa pudo parecerme, a primera vista, un ejercicio fácil. En los últimos catorce años, este texto -0 , más bien, mi propia versión re-escrita para la docencia - forma parte de esos referentes clave que entiendo que los/as estudiantes de Geografía Política han de conocer. He explicado el concepto de área nuclear ${ }^{1}$ aplicado a los Estados europeos al menos dos veces por curso académico, y la totalidad del modelo ha sido la base para presentar en el aula dos cuestiones: por un lado, el contexto de la Geografía Política como disciplina académica en los años 1960; es decir, la ausencia de la disciplina en el contexto académico de la Europa continental, como resultado de una confusa identificación de la Geografía Política con la Geopolítica (parte de la Geografía Política) con la Geopolitik (argumentario de base geopolítica desarrollado en el marco de las políticas del Tercer Reich en la Universidad de Munich)². La Geografía Política estuvo relegada al ámbito anglosajón durante unos treinta años, centrada en produ-

\footnotetext{
${ }^{1}$ Mantendré la traducción de core-area por “área nuclear”, utilizada en la traducción del libro de Geografía Política de Taylor y Flint (2002). Otros autores han propuesto traducir el concepto como "región focal" (López Trigal, 2013: 259), enfatizando esa capacidad de nuclear espacios en torno a un foco que Pounds y Ball proponen como eje de los Estados europeos. Sin embargo, y por razones puramente sentimentales derivadas del trabajo docente de una década, he decidido seguir con las áreas nucleares.

${ }^{2}$ Ver Cairo (2012).
} 
cir análisis donde examinar los factores políticos como parte de los procesos espaciales no era prioritario.

Por otro lado, este texto me ayuda a transmitir algunas generalidades propias del enfoque desarrollista; como perspectiva de análisis, el desarrollismo tiene una presencia muy significativa no sólo en Geografía Política, sino en ciencias sociales en general, desde los años 1960. Desde estas dos premisas, y a partir del mapa de las áreas nucleares de los Estados europeos, trabajar con el texto de Pounds y Ball ha sido fácil. Además de por la sencillez del modelo, porque sirve para tratar otras cuestiones, como la inevitabilidad de los Estados (desde Tilly) o como la difícil aplicación del prototipo fuera de Europa (desde Taylor).

Sin embargo, comentar este artículo de geografía histórica de Europa requeriría algo más. No sólo un comentario algo más intenso del texto, sino también una lectura en perspectiva, no sólo por la renovación de la Geografía Política que comenzó a principios de los años 1970, sino también por los cambios en las formas de comprender la carrera académica de los últimos cincuenta años. Para ello, comenzaré con una breve semblanza de la trayectoria de Norman Pounds ${ }^{3}$.

\section{El autor}

Norman John Greville Pounds falleció en 2006, a los 94 años. Según un obituario publicado en The Guardian ${ }^{4}$, hasta 2005 visitaba cada día la biblioteca de la Universidad de Cambridge en un vehículo eléctrico para personas con movilidad reducida, e impartía docencia en la universidad para mayores, donde sus clases siempre estaban entre las más populares 5 .

De hecho, terminó su carrera en el mismo lugar donde la comenzó. Estudió geografía e historia, en el Fitzwilliam College de Cambridge, con maestros como George Gordon Coulton, especializado en historia medieval y también en visitar con sus estudiantes las iglesias de Cambridge para inspeccionar la mampostería y mobiliario religioso en busca de registros para la investigación. Después de doctorarse con una tesis sobre la geografía histórica de Cornualles que realizó con una beca de investigación en la London School of Economics, Norman regresó a Cam-

\footnotetext{
${ }^{3}$ Resulta imposible seguir el rastro académico o encontrar información alguna sobre la coautora del texto, Sue Simons Ball.

${ }^{4}$ Ver URL: <http://www.theguardian.com/news/2006/apr/13/guardianobituaries.obituaries>, consultado el 25 de noviembre de 2014.

${ }^{5}$ Pese a tener una extraordinaria devoción por la docencia, no tenía reparo en suspender a toda una clase, resuelto a enseñar escritura de investigación en geografía regional en la época de la revolución cuantitativa (Black, 2010).
} 
bridge en 1944, a causa de la evacuación de la escuela fruto de la evolución de la Segunda Guerra Mundial.

En 1949 acepta una plaza de profesor visitante en la Universidad de Wisconsin, lugar de trabajo de geógrafos políticos como Richard Hartshorne, Yi-Fu-Tuan o, más recientemente, Robert Sack ${ }^{6}$ Y un año después, se incorpora a la Universidad de Indiana, en Bloomington, donde se jubilará, en 1977, como catedrático de Geografía e Historia (Black, 2010: 2). Una vez más, regresa a Cambridge desde donde desarrollará tareas como las de presidente de la Royal Archaeological Institute (1987-1990) y de la Cambridge Antiquarian Society (1992-1994).

Sus investigaciones se centraron en explorar la geografía histórica y económica de Europa; y, aunque en sus obituarios se hace referencia a su buen humor, y al gusto por la música de Beethoven y por practicar rugby, también se menciona su capacidad de trabajo sobresaliente y su excepcional dedicación a la docencia y también a la investigación. Publicó más de 30 libros, entre los que destacaría, además de Political Geography (1963), The Ruhr: A Study in Historical and Economic Geography (1952), Heart and Home: a history of material culture (1989) ${ }^{7}$, An Historical Geography of Europe —en tres volúmenes (1977, 1980, 1985) que reescribió en uno solo, publicado en $1990^{8}$ — o The medieval city (2005). En cualquier caso, la que es considerada su obra más importante se publicó en el año 2000, veintitrés años después de su jubilación, con el título de A History of the English Parish. A día de hoy, ésta sigue siendo un referente para investigaciones interdisciplinares, al proponer una aproximación a las parroquias inglesas como formaciones culturales populares, distinguiendo entre sus funciones seculares y religiosas, pero subrayando su función clave en la organización social.

Pounds también publicó decenas de artículos en revistas académicas; es el caso del texto que nos ocupa, publicado con Sue Simons Ball en los Annals of the Association of American Geographers, en 1964.

\section{El artículo}

Como decía en la anterior sección, la investigación y la docencia de Ball se centraron fundamentalmente en cuestiones de geografía histórica; y, precisamente, es una lectura histórica de transformaciones espaciales en el contexto europeo el objeto de “Áreas nucleares y el desarrollo del sistema de Estados europeo". El propósito del

\footnotetext{
${ }^{6}$ De hecho, Pounds reseñó Human Territoriality. Its theory and history, de Sack, para The Journal of Modern History, en 1989.

${ }^{7}$ Traducido al castellano como La vida cotidiana: historia de la cultura material (Barcelona: Crítica, 1999).

${ }^{8}$ Este volumen se tradujo al castellano con el título de Geografía Histórica de Europa (Barcelona: Crítica, 2000).
} 
texto es examinar el proceso de crecimiento territorial de los Estados en Europa a través de la aplicación del concepto de área nuclear, que los autores definen como:

En términos de la Edad Media, que es el periodo del que se ocupa este artículo, incluye una tierra fértil, bien cultivada dentro de los límites de la tecnología moderna, una población lo suficientemente densa como para extraer la máxima ventaja de los recursos locales y, de forma general, un comercio de larga distancia que permita obtener los materiales no disponibles a nivel local (Pounds y Ball, 1964 [2014: 100]).

Esta área, entonces, se convertiría en motor del crecimiento territorial del Estado moderno, cuestión que exploran con detenimiento para el caso de veinticinco Estados europeos. Estos Estados son clasificados en dos tipos: por un lado, lo que denominan Estados arbitrarios (Pounds y Ball, 1964 [2014: 99]), o aquellos Estados creados para demarcar políticamente un marco territorial previamente desarrollado (Pounds y Ball, 1964 [2014: 99]). Y, por otro, los Estados orgánicos (Pounds y Ball, 1964 [2014: 99]), esto es, aquéllos que han crecido en torno a un área germinal, y que serían el prototipo de explicación del crecimiento territorial de los Estados desde la agregación de espacios a un área nuclear. Este sería el caso de quince de los veinticinco Estados examinados.

Es interesante destacar que el paradigma del modelo es el caso de la creación del Estado francés (Pounds y Ball, 1964 [2014: 102-104]. La forma final del Estado moderno, en este caso, se debería a una extensión de límites territoriales ligados a cuestiones políticas (sucesión, guerra, etc.). Parecido sería el caso de Gran Bretaña; aunque, curiosamente, la localización del área nuclear, en este caso, se desplaza entre versiones del modelo. De hecho, en la primera formulación del planteamiento, publicada por Pounds como autor único en 1963 en un manual de Geografía Política, situaba el área nuclear en Escocia y no en el gran Londres, donde queda fijada en la versión que estamos trabajando ${ }^{9}$. Sin embargo, en este artículo, la inclusión de la anexión de Escocia a través de la unión parlamentaria en 1707 sería lo que probablemente explique la relocalización del área en el valle del Támesis. En cualquier caso, es interesante destacar que los autores consideren los procesos de formación del Estado en Francia y en Gran Bretaña como paradigmáticos, en términos de funcionamiento del modelo. En línea con los horizontes normativos ligados al pensamiento desarrollista, estos dos casos han sido considerados frecuentemente como medida y también como pauta de evaluación de diferentes procesos de desarrollo.

A los casos de Francia y Gran Bretaña, se van añadiendo los análisis de Irlanda, Suiza, Suecia, Noruega, Finlandia, Dinamarca, Checoslovaquia, Austria, Hungría,

\footnotetext{
${ }^{9}$ Ver Taylor y Flint (2002: 164).
} 
Rusia, Rumania, Italia y Grecia. Con excepción de Suiza, todos estos Estados presentarían un área nuclear con ciertos elementos comunes. Sin embargo, alguno de estos casos puede servir para exponer una de los elementos que entiendo son claves a la hora de analizar las construcciones estatales. En el caso de Italia, los autores sostienen la idea de una primacía ideológica de Roma sobre las ciudades comerciales del norte del país para justificar su carácter de área nuclear (Pounds y Ball, 1964 [2014: 115-116]). Esta primacía, superviviente de la época del Imperio romano, estaría relacionada con la cuestión de imaginarse políticamente como una forma política unida. Y sí, ciertamente, las decisiones políticas y la imaginación espacial de una entidad delimitada son claves para contextualizar los Estados modernos en Europa. Las características de las áreas nucleares, en sí mismas, pueden ser motores de crecimiento; pero también pueden no serlo, como en el caso de las ciudades del norte italiano. Sería interesante ver qué habría ocurrido si no existiese una proyección política de expansión o de cambio territorial ligado a un proyecto político, paradigmático, por ejemplo, de la unificación italiana del siglo XIX. Como argumenta Mann (2006) la centralización territorial es fundamental para entender el Estado moderno, pero esa centralización territorial tiene lugar dentro de una lógica ligada a los cambios políticos, económicos y sociales que venían ocurriendo desde el final de la Edad Media. El surgimiento de la soberanía territorial a partir del reconocimiento de los límites territoriales de las unidades políticas que se da con la firma de la paz de Westfalia respondería a una suerte de primer concierto de Europa, después de que las formas de hacer la guerra comienzan a requerir de una formalización en la movilización de recursos (Mann, 2006); después de que las políticas aduaneras propias del primer mercantilismo en parte de los Países Bajos comiencen a mostrarse como efectivas para incrementar los beneficios comerciales y "la acumulación de riqueza en un territorio" (Taylor y Flint, 2002: 175). Y después de que las cambiantes alianzas entre la naciente burguesía, la aristocracia y las dinastías monárquicas definiese áreas de desarrollo político y económico más allá de las lealtades personales propias del régimen anterior. En mayor o menor medida, la forma territorial de un Estado moderno territorialmente compacto está ligada a cuestiones políticas, económicas y sociales contextuales sin las que, como aluden Pounds y Ball para el caso de Italia y de la nuclearidad de Roma, es complicado comprender tanto la transformación de las espacialmente dispersas identificaciones políticas medievales como la topología de la articulación territorial de los Estados modernos.

En un segundo nivel de análisis, los autores aplican el prototipo del modelo a España, Portugal, a la desaparecida Yugoslavia y a Bulgaria (Pounds y Ball, 1964 [2014: 119-121]. En todos estos casos, el factor común sería que las áreas nucleares en torno a las cuales estos Estados se habrían articulado originalmente habrían pasado a ser periféricas, marginales en su significación. Y aquí sería interesante de nuevo comentar un caso, el de España. La "nuclearidad" del norte de la actual Castilla y León, o de la zona transfronteriza del sur de Galicia y norte de Portugal 
en la construcción del Estado español es, cuanto menos, cuestionable; pero, lo que resultaría más interesante es que no se refleje en modo alguno la nuclear importancia del área donde se situaba el centro de toma de decisiones políticas sobre el proyecto de creación de ese Estado. Así, ahondando en lo ya mencionado anteriormente, el factor político contextual se torna secundario en un modelo geográficopolítico; en este caso, ocurre lo contrario que lo enunciado por los autores para el caso de Roma.

Por último, en un tercer grupo de Estados, se alude a los casos de Albania, Andorra, Bélgica, Luxemburgo, Liechtenstein, Mónaco, San Marino y los Países Bajos, considerados como ejemplos de formaciones territoriales que no se habrían construido en torno a un área nuclear (Pounds y Ball, 1964 [2014: 122]. En la tendencia general del modelo, estos Estados son considerados excepciones, ya que serían resultado de "un acto creativo imprevisto" (Pounds y Ball, 1964 [2014: 125]). Y es precisamente desde este tipo de argumentación donde el modelo redunda en una tendencia general que ya anticipábamos: la cuestión política en la construcción estatal tiene un papel menor. De hecho, el proceso de formación de los Estados territoriales en Europa es presentado como final de un proceso ineludible, resultado de un desarrollo territorial autónomo, con o sin un área nuclear central, y con los mismos resultados, esto es, la creación de un Estado. Esta inevitabilidad de las formaciones estatales ya ha sido magistralmente cuestionada por Tilly (1992) o por Hechter y Brustein (1980), por seguir en la sociología histórica; por geógrafos políticos (Agnew, 2005) o por geógrafos históricos (Sack, 1986). La consideración de la propia razón de Estado (política) en la construcción de los Estados, en el contexto de las tendencias propias del final de la Edad Media, serían, a mi juicio, los elementos necesarios para continuar profundizando en el conocimiento de los procesos de creación de identidades político-territoriales en Europa sin caer en una suerte de fetichización espacial ${ }^{10}$.

Por último, cabe comentar el resultado de la extensión del argumento del artículo a la explicación de la unidad y cohesión territorial actual, o, en este caso, con la referencia temporal de 1964 (Pounds y Ball, 1964 [2014: 126]. Aunque es una línea de investigación simplemente esbozada por los autores, sería importante desarrollar un escrutinio algo más detallado a la hora de generalizar ese planteamiento, ya que la integración o desintegración territorial tienen una considerable lectura política; en

\footnotetext{
${ }^{10}$ De hecho, una de las fortalezas de este artículo es precisamente esa, que se centra en el contexto europeo, como escenario en el que se va construyendo, imaginando y negociando la formación político-territorial del Estado. Ese formato estatal se mantiene para la incorporación de las colonias a la comunidad internacional como sujetos políticamente independientes, con los procesos de independencia, pero es un producto europeo. En la exposición de este texto, Taylor y Flint (2002: 164) hacen referencia a la aplicación del modelo fuera de Europa, que mostraría la vinculación entre el desarrollo de áreas nucleares y los procesos políticos de colonización e imperialismo. Sin embargo, en este texto, el contexto es europeo.
} 
otras palabras, la politización de los factores espaciales ligados a procesos de cohesión de un Estado van más allá de un proceso histórico de crecimiento territorial.

Ahora bien, aunque el modelo pueda adolecer de una cierta falta de lectura política, o, dicho de otra manera, de una distancia del espacio y tiempo político ligados a la creación del Estado moderno como forma política ${ }^{11}$, es necesario señalar, precisamente, el espacio y el tiempo de producción del propio texto. El artículo es un ejercicio riguroso y ambicioso de sistematización de explicar tendencias y conceptos propios de la sociedad moderna, desde una propuesta de modelo explicativo.

Trabajar sobre la evolución de las áreas nucleares en Europa, incluyendo constantes referencias a la geografía física, la historia social o las formaciones culturales, es algo propio de formaciones amplias, interdisciplinares y vinculadas con el tiempo necesario para producir conocimiento. La incremental especialización ligada a los cambios en la valoración de currículums, unidas a las crecientes cargas docentes y a los imposibles requisitos de valoración de la investigación, complica considerablemente las tareas de artesanía argumentativa y de puesta en valor de diferentes perspectivas y pequeños registros necesarios para construir propuestas de análisis amplias e incluso generalizables. Recabar datos de los censos parroquiales para trabajar sobre la peste negra, objeto de uno de los múltiples manuscritos inacabados de Pounds, es una tarea que se desarrolla desde la inquietud, y se mantiene por vocación, pero que se alimenta sólo si hay un tiempo para enseñar, un tiempo para producir, y un espacio para reflexionar sobre cuestiones que requieren de una temporalidad reposada. En ese espacio, leer a Pounds resulta intelectualmente reconfortante.

\section{Bibliografía}

Agnew, J. (2005) Geopolítica: una re-visión de la política mundial. Madrid: Trama [Trad. por M. Lois del original: Geopolitics: Re-visioning World Politics. Londres: Routledge, 2003, $2^{\mathrm{a}}$ ed.].

Black, W. (2010) "Memorial Resolution Professor Emeritus Norman J.G. Pounds", Universidad de Indiana en $<$ http://www.indiana.edu/ bfc/docs/circulars/09-10/B22-2010.pdf >, consultado el 25 de noviembre de 2014].

Cairo, H. (2012) "Comentario. La Geopolítica como «ciencia del Estado»: el mundo del general Haushofer". Geopolítica(s). Revista de estudios sobre espacio y poder, vol. 3, núm. 2, 2012, 337-345.

\footnotetext{
${ }^{11}$ Sería algo visible también en la constante utilización de la palabra "Estado" para referirse a formas de organización política premodernas.
} 
Hechter, M., y Brustein, W (1980) "Regional modes of production and patterns of state formation in Western Europe”. American Journal of Sociology, vol. 85, núm. 5, 1061-1094.

López Trigal, L. (2013) Diccionario de Geografía Política y Geopolítica. León: Universidad de León.

Mann, M. (2006) "El poder autónomo del Estado: sus orígenes, mecanismos y resultados”. Revista Académica de Relaciones Internacionales, núm. 5, 1-43.

Pounds, Norman J., y Ball, Sue Simons (2014) "Áreas nucleares y el desarrollo del sistema de Estados europeo”. Geopolítica(s). Revista de estudios sobre espacio y poder, vol. 5, núm. 1, 99-126. [Trad. del original por M. Díaz: "Core-Areas and the Development of the European States System", Annals of the Association of American Geographers, vol. 54, núm. 1, 1964, 24-40].

Sack, R. D. (1986) Human Territoriality: Its Theory and History, Cambridge: Cambridge Studies in Historical Geography.

Taylor, P. J., y Flint, C. (2002) Geografía Política: Economía-mundo, Estadonación y Localidad. Madrid: Trama Editorial ( $2^{\mathrm{a}}$ ed. en castellano) [Trad. por H. Cairo y A. Despujol del original: Political geography: World-Economy, NationState and Locality. Londres: Pearson Education, 2000, $\left.4^{\mathrm{a}} \mathrm{ed}.\right]$.

Tilly, C. (1992) Coerción, capital y los Estados europeos, 990-1990. Madrid: Alianza. 